\title{
A preliminary investigation of genetic counselors' information needs when receiving a variant of uncertain significance result: a mixed methods study
}

\author{
Courtney L. Scherr, PhD ${ }^{1}$, Noralane M. Lindor, MD², Teri L. Malo, PhD, MPH'1, \\ Fergus J. Couch, $\mathrm{PhD}^{3}$ and Susan T. Vadaparampil, PhD, $\mathrm{MPH}^{1}$
}

Purpose: The aim of this study was to explore genetic counselors information preferences on reports of variant of uncertain significance (VUS) results from cancer genetic testing.

Methods: This mixed methods report (quantitative and qualitative approaches) utilized a survey of genetic counselors containing closedand open-ended questions to explore genetic counselors' information needs and perceptions of the industry's current information sharing practices. Descriptive statistics were calculated for responses to the closed-ended questions, and thematic analysis guided the interpretation of the open-ended questions.

Results: Of the 267 participants (28.6\% response rate), the majority indicated a perceived lack of information on VUS laboratory reports, were concerned about the perceived practice of withholding information, and stated the information they wanted to see. Although most did not indicate how additional information would be used, some reported they would provide information directly to patients, and others reported that the information would be used to contextualize the VUS result when counseling patients.

Conclusion: This analysis identified information that genetic counselors believe is needed in VUS reports, indicating what they believe are best practices in lieu of guidelines for laboratories currently providing genetic testing services. Future studies should explore how genetic counselors use additional information contained in VUS reports.

Genet Med advance online publication 8 January 2015

Key Words: genetic counseling; genetic testing; hereditary breast and ovarian cancer; information needs; variant of uncertain significance

\section{INTRODUCTION}

Approximately $5-10 \%$ of women with breast cancer ${ }^{1}$ and $15 \%$ of women with ovarian cancer ${ }^{2}$ have a mutation in the BRCA1 or BRCA 2 (BRCA) genes. By the age of 70 years, women with a $B R C A 1$ mutation have a $60 \%$ average risk of developing breast cancer and a $59 \%$ average risk of developing ovarian cancer, and women with a BRCA2 mutation have a $55 \%$ average risk of developing breast cancer and a $16.5 \%$ average risk of developing ovarian cancer. ${ }^{3}$ Definitive genetic test results (i.e., true positive or true negative) can guide prevention, diagnosis, and treatment to reduce cancer incidence and mortality. ${ }^{4-6}$ Unfortunately, genetic test results are not always straightforward. For example, a variant of uncertain significance (VUS) result indicates a change in a $B R C A$ gene was detected, yet the conferred cancer risk resulting from that change is unknown. This result is among the most difficult to discuss with patients. ${ }^{7.8}$

Ongoing efforts to reclassify VUS rely on laboratory-based and/or epidemiological studies to generate sufficient data to determine the likelihood a gene mutation is harmful (i.e., pathogenic or deleterious). ${ }^{9}$ Until these studies yield clinically relevant findings, genetic counselors (GCs) will face challenges associated with disclosing VUS results to patients. Yet, to our knowledge, only one prior US-based study examined GC practices related to VUS, ${ }^{7}$ and none examined the information needs of GCs, for example, the amount and type of information included on laboratory reports and preferences for receiving updates when VUS are reclassified.

As part of a larger study to optimize the integration of VUS reclassification data into clinical practice, a national survey of GCs was used to explore attitudes, practice patterns, and information preferences related to BRCA VUS reporting and counseling. The purpose of the current study was to analyze responses from closed- and open-ended questions using a mixed methods approach to identify information needs of GCs related to VUS reports and updates.

\section{Recruitment and data collection \\ MATERIALS AND METHODS \\ Mailing lists from the National Society of Genetic Counselors (NSGC) and the American Board of Genetic Counseling were used to identify GCs who practiced cancer genetic counseling, reported clinical practice as their primary activity, and had a}

${ }^{1}$ Health Outcomes and Behavior Program, H. Lee Moffitt Cancer Center, Tampa, Florida, USA; ${ }^{2}$ Department of Health Sciences Research, Mayo Clinic, Scottsdale, Arizona, USA; ${ }^{3}$ Department of Laboratory Medicine and Pathology, Mayo Clinic, Rochester, Minnesota, USA. Correspondence: Susan T. Vadaparampil

(Susan.Vadaparampil@moffitt.org) 
US mailing address. The NSGC provided a spreadsheet of all members $(n=2,451)$ containing information about practice, specialization, and work setting. This list was refined to include only members who listed "cancer" or "personalized genomic medicine" as their primary or other specialization; listed "cancer" as an area of practice, including "cancer genetics," "adult cancer genetics," and "cancer risk counseling;" and/or who worked at an organization with "cancer" or "oncology" in the name. This resulted in a list of 751 GCs. The American Board of Genetic Counseling provided a presorted mailing list of 621 members practicing cancer genetic counseling in the United States. The lists were merged and duplicates (i.e., individuals listed on both) were removed, resulting in a list of 975 GCs. An additional 43 GCs were removed, including 11 who piloted the survey for face and content validity and those identified as duplicates during the mailing process, resulting in a total sample of 932 GCs.

After institutional review board approval was obtained from Mayo Clinic and the University of South Florida, a multiphase recruitment approach ${ }^{10}$ was utilized. GCs were mailed a postcard to alert them to the upcoming survey and to verify the accuracy of mailing addresses. Seven weeks later, a packet containing a cover letter, survey, prepaid return envelope, prepaid response card, and two articles about VUS reclassification ${ }^{11,12}$ was mailed. The prepaid response card allowed GCs to indicate survey completion and enter a drawing for one of four $\$ 50$ bills. Surveys were mailed in February 2013; completed surveys were accepted through 15 May 2013.

Surveys can be an efficient and cost-effective approach to gather data among a national sample of providers; however, respondents are limited by the set of predetermined fixed responses. Including free-text areas on surveys may allow other attitudes and beliefs to emerge, providing additional insight into important factors to address the needs of health-care providers. ${ }^{13}$

Quantitative data collection and analysis. To capture GCs' perceptions about the amount of information included on BRCA VUS reports, participants selected one of three response options to the following statement: "In your opinion, the amount of information currently provided by Myriad Genetic Laboratories about BRCA VUS results is: too much, just right, or too little." (This survey was conducted prior to the court decision that led to multiple laboratories offering $B R C A$ testing; at the time of this survey, Myriad Genetics was the sole provider for BRCA testing in the United States.) GCs were asked to indicate which resources they used and how often when VUS results were received, as well as which guidelines they referred to and how often when counseling patients with VUS results (see Table 1 for response options). GCs were asked to indicate the clinical screening they recommend for patients based on receiving a VUS test result. Response options included: counsel as if a positive result (full high-risk surveillance); counsel based on family history and other risk factors; or other. Finally, GCs were asked to indicate the preferred source for receiving professional education and information on VUS reclassification (see Table 2 for response options) and preferred channel of communication (see Figure 1 for response options).

Frequencies and percentages for participant demographics and responses to the aforementioned questions were calculated using SAS 9.3.

Qualitative data collection and analysis. At three points in the survey, respondents were asked to provide written comments. The first open-ended question(Q1) was a follow-up to the closedended question about the amount of information currently included on BRCA VUS reports and asked, "If you answered too much or too little to the question, please explain." The other qualitative questions on which this study draws appeared at the end of the survey: "What additional information would you like to have available on $B R C A$ VUS to help you counsel your patients" (Q2); and "If you have any additional comments you would like to share with our research team, please feel free to share them here"(Q3). Thematic analysis was used to identify themes in responses. Three coders independently reviewed all responses to each question and completed rounds of open coding, creating categories, and abstraction. ${ }^{14}$ Coders met after

Table 1 Resources and sources currently used to counsel patients about variants of uncertain significance $(N=267)$

\begin{tabular}{|c|c|c|c|}
\hline Resource & $\begin{array}{c}\text { Never/rarely } \\
n(\%)^{\mathrm{b}}\end{array}$ & $\begin{array}{c}\text { Sometimes }^{\mathrm{a}} \\
n(\%)^{\mathrm{b}}\end{array}$ & $\begin{array}{c}\text { Often/always } \\
n(\%)^{\mathrm{a}}\end{array}$ \\
\hline Contact Myriad Genetics & $25(9.3)$ & $62(23.2)$ & $179(67.1)$ \\
\hline Search scientific literature & $60(22.5)$ & $58(21.7)$ & $147(55)$ \\
\hline Search Breast Information Core website & $145(54.3)$ & $41(15.4)$ & $78(29.2)$ \\
\hline Discuss with a colleague & $29(10.9)$ & $51(19.1)$ & $181(67.8)$ \\
\hline NSGCc & $43(16.1)$ & $62(23.2)$ & $157(58.8)$ \\
\hline $\mathrm{NCCN}^{\mathrm{d}}$ & $20(7.5)$ & $23(8.6)$ & $219(82.1)$ \\
\hline IARC & $196(73.4)$ & $43(16.1)$ & $19(7.1)$ \\
\hline
\end{tabular}

aResponse options were based on a 5-point Likert-type scale from never to always. ${ }^{b}$ Percentages may not sum to 100 due to missing data. cNational Society of Genetic Counselors (NSGC), http://www.nsgc.org. 'National Comprehensive Cancer Network (NCCN), http://www.nccn.org. eInternational Agency for Research on Cancer (IARC), http://www.iarc.fr. 
each coding phase to discuss and compare results and resolve differences in coding, categorization, and abstraction.

Initially, responses to each question were coded independently, for example, coding was completed on all responses to Q1 separately from Q2 and Q3. However, significant overlap in responses and themes to each question was identified; therefore, responses from all three questions were analyzed together to achieve a holistic understanding of GCs' views regarding VUS results. The resulting themes were drawn from responses to all three questions.

\section{Participants}

\section{RESULTS}

A total of 410 completed surveys were received. Because the survey was designed for individuals with experience providing genetic counseling for hereditary breast and ovarian cancer, 12 participants were excluded because they reported no experience providing genetic counseling for hereditary breast and ovarian cancer or had missing data. Of the 398 GCs who completed the survey (42.7\% total response rate) and were considered for analysis, approximately 267 (28.6\% response rate) provided written comments to at least one of the three open-ended questions. Only those who provided responses to the open-ended questions were included due to the interrelated nature of this analysis. On comparing those who responded to the open-ended questions with those who did not respond, no statistically significant differences between groups were found for age, sex, race, ethnicity, or board certification (Table 3 ). A Pearson $\chi^{2}$ test showed those who reported cancer as their primary specialty $\left(x^{2}(1, N=396)\right.$ $=16.16, P<0.0001)$ and those who reported providing direct patient care as their primary role $\left(x^{2}(1, N=397)=11.99, P=\right.$ 0.0005 ) were more likely to respond to the open-ended questions.

After removing responses including "n/a," "none," "no," or "0," 239 participant comments were included in the

Table 2 Preferences for professional education $(N=267)$

\begin{tabular}{|c|c|c|c|c|c|}
\hline Source & $\begin{array}{l}\text { Do not } \\
\text { prefer, } n(\%)^{a}\end{array}$ & $\begin{array}{l}\text { Somewhat } \\
\text { prefer, } n(\%)^{a}\end{array}$ & $\begin{array}{l}\text { Neutral, } \\
n(\%)^{\mathrm{a}}\end{array}$ & $\begin{array}{l}\text { Moderately } \\
\text { prefer, } n(\%)^{a}\end{array}$ & $\begin{array}{c}\text { Strongly } \\
\text { prefer, } n(\%)^{a}\end{array}$ \\
\hline Direct communication from Myriad & $7(2.6)$ & $8(3.0)$ & $17(6.4)$ & $68(25.5)$ & $167(62.6)$ \\
\hline Updated reclassification lists mailed & $47(17.6)$ & $20(7.5)$ & $38(14.2)$ & $80(30.0)$ & $79(29.6)$ \\
\hline $\begin{array}{l}\text { Updated reclassification lists posted on Facing Our } \\
\text { Risk of Cancer Empowered website }{ }^{b}\end{array}$ & $110(41.2)$ & $23(8.6)$ & $57(21.4)$ & $53(19.9)$ & $21(7.9)$ \\
\hline $\begin{array}{l}\text { Updated reclassification lists posted on NSGC } \\
\text { website }\end{array}$ & $55(20.6)$ & $20(7.5)$ & $67(25.1)$ & $69(25.8)$ & $55(20.6)$ \\
\hline
\end{tabular}

NSGC, National Society of Genetic Counselors.

aPercentages may not sum 100 due to missing data. ${ }^{b}$ Facing Our Risk of Cancer Empowered, http://www.facingourrisk.org.

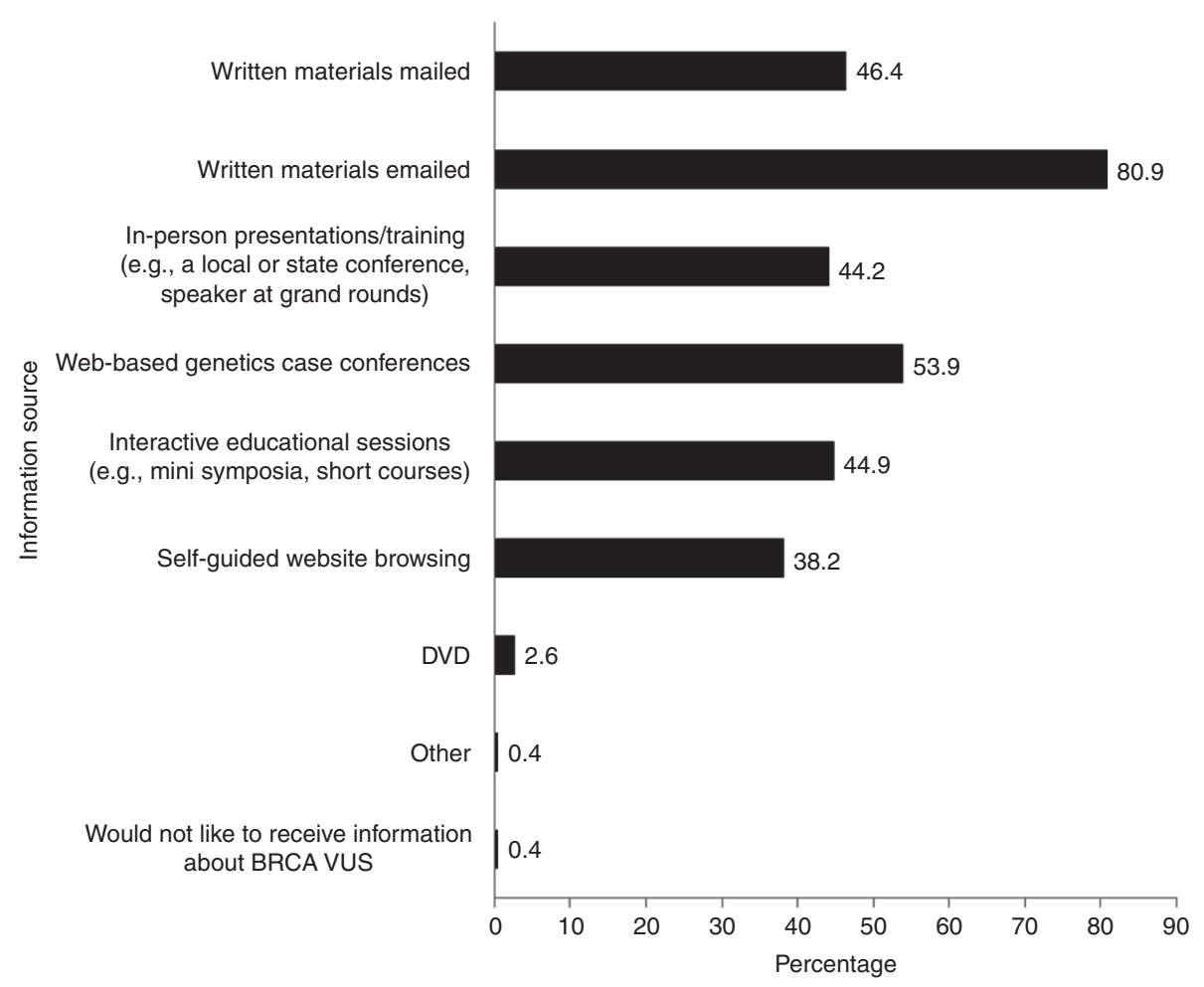

Figure 1 Preferred format for professional education/information $(N=267)$. 
Table 3 Comparison of samples, stratified by participants who did versus did not provide comments

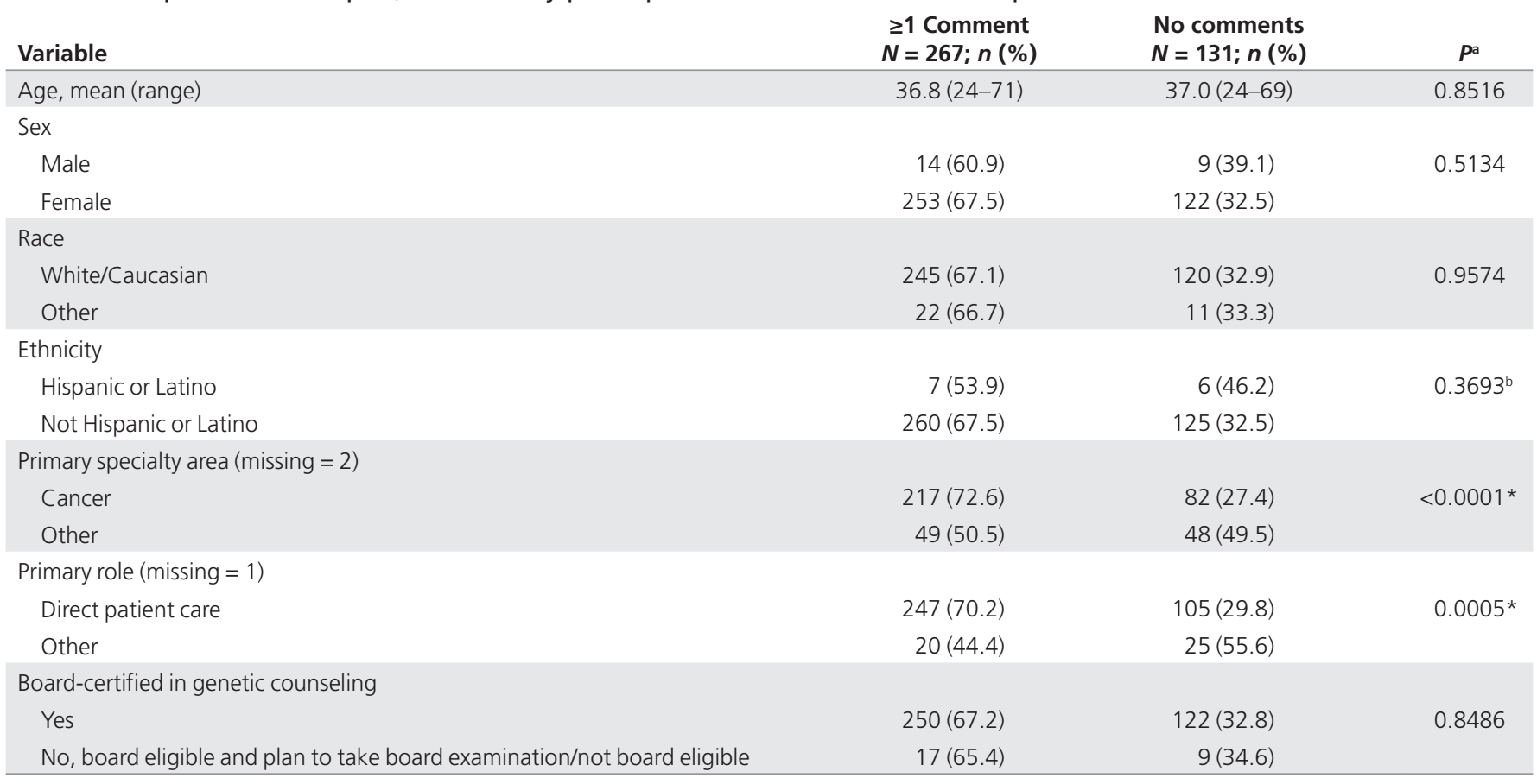

Row percentages are reported.

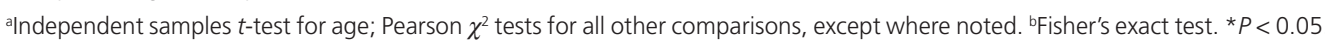

qualitative analysis from Q1, 109 were included from Q2, and 46 were included from Q3. Participants had provided genetic counseling for hereditary breast and ovarian cancer for an average of 7.4 years and were predominately female $(94.8 \% ; n=253)$, Caucasian $(91.8 \% ; n=245)$, and non-Hispanic $(97.4 \% ; n=260)$. The primary specialty for the majority was cancer $(81.3 \% ; n=217)$, and the majority indicated their primary role was direct patient care $(92.5 \% ; n=247)$ and were board certified in genetic counseling $(93.6 \% ; n=$ 250; Table 3).

\section{Quantitative results}

Of respondents, 243 (91\%) reported the amount of information provided on BRCA VUS laboratory reports was too little, $1(0.4 \%)$ reported too much, and 23 (8.6\%) reported just enough. When VUS results were received, the majority reported often or always using supplemental reports provided by the laboratory $(67.8 \%)$ or discussing results with a colleague (67.8\%), followed closely by contacting the laboratory directly $(67.1 \%)$ and searching the scientific literature (55\%; Table 1). When faced with a VUS, guidelines from the National Comprehensive Cancer Network (NCCN) (55.1\%) guided practice most frequently, followed by guidelines from the NSGC $(22.5 \%)$ and the International Agency for Research on Cancer (7.1\%; Table 1). When counseling patients with a VUS, 254 (95.1\%) reported providing clinical guidelines based on family history, whereas $6(2.3 \%)$ reported counseling as if the result was positive and $5(1.9 \%)$ reported "other."
The majority strongly preferred to receive reclassification information about BRCA VUS through direct communication from the laboratory about their patient only $(62.6 \%)$, followed by comprehensive lists of reclassified VUS mailed to them directly (29.6\%), posted on NSGC website (20.6\%), or posted on the Facing Our Risk of Cancer Empowered website $(7.9 \%$; Table 2$)$. The preferred delivery of information was written materials sent via e-mail (80.9\%), followed by a Web-based genetics case conference (53.9\%), mailed written materials (46.4\%), an interactive educational session (44.9\%), in-person presentations/trainings (44.2\%), or a self-guided website (38.2\%). A minority preferred a DVD (2.6\%) and one participant did not want to receive information about $B R C A$ VUS (0.4\%; Figure 1).

\section{Qualitative results}

GCs would like more information about VUS. Consistent with quantitative findings, participants' comments reflected the desire for more information on BRCA VUS laboratory reports. For example, one respondent wrote, "I would like more information about why it is a VUS; they provide almost no information other than ethnicity." In addition, participants listed specific information they preferred on VUS reports, including "prediction models" or "probability models" such as the "polyphen score," "in silico analysis," or "functional data;" "conservation of the sequence;" "co-segregation studies;" "frequency of tracking in families;" "evolutionary conservation data;" "estimated time to reclassification;" and "references to 
any published literature available on VUS." Some mentioned one or two of the aforementioned items; others provided a comprehensive list.

In addition to listing specific desired information, there were references to $B R C A$ VUS reports previously delivered by the laboratory providing $B R C A$ testing at the time of this study and examples of non-BRCA VUS reports from other laboratories. For example:

"I do understand their reasoning, but I wish Myriad still provided all the info they previously did regarding the number of times the VUS had been seen and how it tracked in families. I appreciated having that info and find that I do miss it. Also, other labs (doing testing for other genes) provide the in silico model results which could also be helpful in counseling."

GCs proactively sought additional information. In response to the absence of information, participants described proactive methods for acquiring more information, including "calling the laboratory directly," "seeking help from others," and "researching." However, limitations to seeking additional information were also noted, including a "lack of time" and "not much luck." Participants reported complete information was difficult to obtain even when calling the laboratory directly, for example: "Many times it [the VUS report] only includes very vague descriptions and I need to call a genetic counselor at Myriad to get the complete information they have. They often reference "internal data" on families which is so very vague and unfortunate." Some suggested consulting with other providers, as one counselor noted: "Seeking help from other providers is essential for providing proper risk assessment for individuals with a VUS. You can bounce ideas off them, use their past experience, and learn tricks of the trade for dealing with these patients. I highly recommend that anyone who deals often with VUS find some way to get themselves involved with other cancer counselors to discuss these patients, especially as panel tests become more mainstream."

As noted, the anticipated increasing frequency of VUS results arising from the testing of even rarer genes on newly available multigene panels will require GCs to develop strategies to deal with VUS results.

A minority did not want additional information. Although the majority desired more information on the laboratory reports, it is worth noting that a minority reported the information was just right, for example, "Mutation classification (interpretation) should always be the responsibility of the lab doing the test. No genetic counselor or other clinician should ever determine for themselves the clinical impact of the mutation." There was concern additional information could lead GCs to alter medical management based on personal interpretation. It was suggested that the amount of information provided could be tailored according to professional training and clinical setting. For example, "For nongenetics providers, the current amount is just right. However, academic medical centers with genetics/research expertise should have access to additional info."

Transparency. GCs believed testing laboratories had additional information but did not provide it: "I feel that Myriad does not provide enough information about their classification system and about the data they have about their VUS." Participants reported the testing laboratory cited "internal data" when additional information was requested, and the lack of information created a negative feeling toward the organization, "The information is just not helpful because it's not enough detail which is frustrating because Myriad has the info." In addition, the lack of data led GCs to question the laboratory's decision to classify a mutation as a VUS, "We do not have any proof to their classification if nothing has been previously published."

Lack of publicly available shared data. In response to perceived lack of transparency, participants suggested using a publicly available database for information sharing: "It would be nice to have a world-wide collaboration of VUS and not be directly dependent on only the information that Myriad is able to collect." Some cited the Breast Information Core (BIC) as a publicly available resource for information on VUS but indicated an absence of information sharing from the laboratory to the BIC, for example: "My understanding is that Myriad does not report to BIC-I think this is a huge disservice to the genetics community in understanding these mutations/ VUS/polymorphisms."

Communication of results to patients, their family, and other providers. Although participants were not directly asked about how they would use additional information, some specified the information would help them when communicating results with patients, patients' family members, and other providers. Some suggested additional information would not be shared directly with patients, but it could be used to contextualize the result, for example: "I preferred when they used to provide data about whether the VUS had been seen with a known deleterious mutation. While we never used it to change the clinical interpretation or shared it with patients it was helpful for discussion of the "gestalt" of the VUS."

In addition to providing more information, some requested patient education materials, for example: "A visual aid regarding the possibility of a VUS for pre-test counseling as well as a visual aid for post-test counseling when a VUS result is received." Despite not asking directly about patient education materials in the open-ended questions, requests were made for this type of information.

The absence of information about VUS on laboratory reports challenged participants' ability to discuss clinical management (i.e., screening and prevention behaviors) and the possibility for family members to participate in research (i.e., Myriad's Variant Classification Program (VCP) during the posttest counseling session. For example: "In most cases, there is not enough 
detailed information about the variant to help guide clinical management. It is also often difficult to encourage the family to participate in the VCP because in the first appointment [with] the genetic counselor I am often unsure who it will be offered to and/or how it may help."

Finally, a minority of participants wanted information about VUS for other health-care professionals due to concerns about awareness of VUS and appropriate medical recommendations: "There is a HUGE lack of understanding of VUS with many physicians. I have had many patients who were wrongly counseled/advised based upon a VUS result (both counseled as deleterious as well as a negative result). This seems to be happening more and more frequently as more physicians without genetic training are ordering $B R C A$ testing by themselves. And as NextGen panels become more frequently ordered, I am very concerned about what will happen with our patients when they go back and discuss with their physicians (even if I have provided accurate counseling)."

Open-ended questions on the survey did not ask about other providers, nor does this concern relate specifically to additional information, but the concern about other providers' interpretations of VUS was raised by participants and highlighted the unease about the use of panel testing and the potential for negative consequences related to VUS results.

\section{DISCUSSION}

Cancer GCs in the United States were surveyed to explore perceptions about information they receive on VUS reports and to identify needs and mechanisms for providing additional information. Because a significant number of respondents provided written responses to open-ended questions, a mixed methods approach to data analysis was utilized.

Most participants believed laboratory reports containing VUS results lacked information, and they described information they wanted on VUS reports; however, it was unclear how additional information might be used. Some indicated additional information would help contextualize the VUS result for patients. For example, a GC might be able to say something like, "we know very little about whether this variant is present in other families" or "there are several studies currently under way to learn more about cancer risk in families who carry this variant." In addition, previous studies reported GCs perceived a lack of patient understanding about $\mathrm{VUS}^{7}$ and documented negative patient outcomes when receiving VUS results, including higher levels of distress about cancer risk, ${ }^{15}$ interpreting the result as a genetic predisposition to cancer, ${ }^{16}$ and engaging in prevention measures not indicated by their result (e.g., prophylactic surgeries). ${ }^{17}$ These potential negative outcomes and GCs' frustrations with their inability to provide the patient with a clear result ${ }^{7}$ may motivate the desire for additional information on laboratory reports. For clarification, future studies should ask GCs how they would use additional information and identify what information would be the most useful to share with patients.
Although most wanted additional information, it is worth noting a minority of respondents did not. Participants believed it could be problematic if additional information is interpreted by GCs to guide patient medical management. Interpretation, they believed, was the responsibility of the laboratory conducting the test, and medical management should be based solely on personal and family history. These beliefs are consistent with current NCCN and NSGC medical management guidelines for patients with a VUS. ${ }^{18,19}$ The potential for GCs to use additional information for interpretation was viewed as concerning if the information was incomplete or if the interpretation incorrect.

In response to the lack of information provided on VUS test results, GCs reported proactively seeking additional information, including consulting with other colleagues, calling the laboratory for more information, and searching the scientific literature. Interestingly, some requested a publicly available database, but only $\sim 30 \%$ reported often or always using the BIC database. In addition to the BIC, participants infrequently mentioned other websites offering publicly available data regarding VUS (not specific to $B R C A$ ), including MAPP-MMR, Align GVGD, PolyPhen, and Leiden Open Variation Database. These databases provide available evidence and results from mathematical models ${ }^{12}$ that synthesize existing evidence to provide likelihood scores that may guide clinical interpretation. However, they are not regularly updated, they provide limited data, and they do not explicitly state whether this information is intended to guide clinical interpretation and medical management. Therefore, requests for public databases, compounded by the low use of the BIC, may indicate that GCs are unaware of these resources, GCs have uncertainty about how to use them or how to interpret the information, or the resources are not meeting the clinical needs of GCs. Future research should examine cancer GCs' awareness of these databases and barriers to use, and should identify strategies or education for GCs to integrate these resources into clinical practice.

In addition, in 2004, the sole laboratory providing $B R C A$ testing stopped contributing its findings to the BIC database $;{ }^{20}$ in 2010, the laboratory stopped including additional information about VUS-such as variant frequency, ethnic groups in which the variant was observed, cosegregation with disease, and whether the variant was identified in a person with a known deleterious mutation-as part of its report. It may be that the company stopped providing detailed VUS reports in anticipation of the loss of its patent. ${ }^{20}$ These practices likely contributed to the perceived lack of transparency noted by participants and created mistrust of the laboratory and the results. In addition, the lack of information sharing adds to the paucity of publically available information about VUS. Several "Free the Data" campaign $\mathrm{s}^{21}$ are currently under way to encourage sharing of deidentified information about VUS. Laboratories that do share their data may have a competitive advantage over those that do not, because their contribution provides open access to the information used to classify a mutation as a VUS. In addition, the majority preferred previously provided information, updates provided by the laboratory regarding their specific 
patients rather than comprehensive lists provided through other organizations, and wanted updates to be provided via e-mail. Therefore, in the future, providing additional information on VUS reports and providing available updates directly to GCs regarding their patients with VUS may offer another competitive advantage to laboratory companies that do so. Whether this is feasible within the current business models of most laboratories remains to be seen, and the legal obligation of testing laboratories is undefined regarding reanalysis or recontact when new information becomes available.

In addition to the impact on the patient, GCs were sensitive to the impact VUS results may have on the family. Testing other family members to see if it cosegregates with disease in the family is one way to gain information about a VUS.2. The American College of Medical Genetics and Genomics and NCCN guidelines recommend follow-up studies to test a limited number of family members for research purposes only to gather additional data for reclassifying a VUS. ${ }^{18,23,24}$ However, if such studies are unavailable, very careful discussions and counseling are necessary to clarify the costs and goals of testing additional family members for a VUS.

These results were based on six closed-ended questions and three open-ended questions, thereby limiting the depth of analysis to the amount of information provided in participants' written comments; however, information in this report will be useful for future research because a number of questions were raised when analyzing the responses. The population in this study included only GCs who provide cancer genetic counseling, and not all GCs provided written comments. Those who provided comments were more likely to indicate their primary role was direct patient care and their primary specialty area was cancer. These findings may reflect resources needed to deliver optimal patient care in the context of $B R C A$ VUS results, but study results cannot be generalized to all cancer GCs.

Although the purpose of the larger study was not focused on the open-ended responses, the high rate of written comments prompted our team to examine participants' responses to identify other important factors regarding reporting of VUS results. ${ }^{13}$ Nearly $70 \%$ of respondents provided additional comments about their preferences and experiences regarding VUS on this survey, indicating VUS results reporting is a highly charged issue among cancer GCs. In the future, the increased use of multigene panel testing will dramatically increase the rate of VUS results. ${ }^{25}$ GCs will progressively face challenges associated with communicating VUS results to patients, their family members, and other providers. Because most counselors turn to the NCCN for guidance when receiving VUS results, it may be useful for the NCCN to provide additional guidelines for medical management of patients with VUS results. Future studies exploring how cancer GCs handle VUS results in terms of interpretation, providing medical management recommendations, and sharing results with the patient and their family remain critical areas for future study.

\section{ACKNOWLEDGMENTS}

This work was supported by the Breast Cancer Spore CA 116201. C.L.S. is supported by the R25 CA 090314 Behavioral Oncology Education and Career Development grant. This work was supported in part by the Survey Methods Core Facility at the H. Lee Moffitt Cancer Center \& Research Institute, a National Cancer Institutedesignated Comprehensive Cancer Center (P30-CA76292). We thank Mary Lou Smith for providing feedback on an earlier draft of the manuscript. We also thank members of the Research Advocacy Network for their input regarding the development of this project.

\section{DISCLOSURE}

The authors declare no conflict of interest.

\section{REFERENCES}

1. Campeau PM, Foulkes WD, Tischkowitz MD. Hereditary breast cancer: new genetic developments, new therapeutic avenues. Hum Genet 2008;124:31-42.

2. Pal T, Permuth-Wey J, Betts JA, et al. BRCA1 and BRCA2 mutations account for a large proportion of ovarian carcinoma cases. Cancer 2005;104:2807-2816.

3. Mavaddat N, Peock S, Frost D, et al.; EMBRACE. Cancer risks for BRCA1 and BRCA2 mutation carriers: results from prospective analysis of EMBRACE. J Natl Cancer Inst 2013;105:812-822.

4. Domchek SM, Friebel TM, Singer CF, et al. Association of risk-reducing surgery in BRCA1 or BRCA2 mutation carriers with cancer risk and mortality. JAMA 2010;304:967-975.

5. Gage M, Wattendorf D, Henry LR. Translational advances regarding hereditary breast cancer syndromes. J Surg Oncol 2012;105:444-451.

6. Grann VR, Patel PR, Jacobson JS, et al. Comparative effectiveness of screening and prevention strategies among BRCA1/2-affected mutation carriers. Breast Cancer Res Treat 2011;125:837-847.

7. Petrucelli N, Lazebnik N, Huelsman KM, Lazebnik RS. Clinical interpretation and recommendations for patients with a variant of uncertain significance in BRCA1 or BRCA2: a survey of genetic counseling practice. Genet Test 2002;6:107-113.

8. Miller-Samuel S, MacDonald DJ, McDonald DJ, et al. Variants of uncertain significance in breast cancer-related genes: real-world implications for a clinical conundrum. Part one: clinical genetics recommendations. Semin Oncol 2011;38:469-480.

9. Iversen ES Jr, Couch FJ, Goldgar DE, Tavtigian SV, Monteiro AN. A computational method to classify variants of uncertain significance using functional assay data with application to BRCA1. Cancer Epidemiol Biomarkers Prev 2011;20:10781088.

10. Dillman DA. Mail and Internet Surveys: The Tailored Design Method. vol. 2. Wiley: New York, 2000.

11. Lindor NM, Goldgar DE, Tavtigian SV, Plon SE, Couch FJ. BRCA1/2 sequence variants of uncertain significance: a primer for providers to assist in discussions and in medical management. Oncologist 2013;18:518-524.

12. Lindor NM, Guidugli L, Wang X, et al. A review of a multifactorial probabilitybased model for classification of BRCA1 and BRCA2 variants of uncertain significance (VUS). Hum Mutat 2012;33:8-21.

13. Esses VM, Maio GR. Expanding the assessment of attitude components and structure: the benefits of open-ended measures. Eur Rev Soc Psychol 2005;1: 71-101.

14. Elo S, Kyngäs H. The qualitative content analysis process. J Adv Nurs 2008;62:107-115.

15. Vos J, Gómez-García E, Oosterwijk JC, et al. Opening the psychological black box in genetic counseling. The psychological impact of DNA testing is predicted by the counselees' perception, the medical impact by the pathogenic or uninformative BRCA1/2-result. Psychooncology 2012;21:29-42.

16. Vos J, Otten W, van Asperen C, Jansen A, Menko F, Tibben A. The counsellees view of an unclassified variant in BRCA1/2: recall, interpretation, and impact on life. Psychooncology 2008;17:822-830.

17. Murray ML, Cerrato F, Bennett RL, Jarvik GP. Follow-up of carriers of BRCA 1 and BRCA2 variants of unknown significance: variant reclassification and surgical decisions. Genet Med 2011;13:998-1005.

18. Daly MB, Pilarski R, Axilbund JE, et al. Genetic/familial high-risk assessment: breast and ovarian, version 1.2014 J Natl Compr Canc Netw 2014;12: 1326-1338. 


\section{ORIGINAL RESEARCH ARTICLE}

19. Berliner JL, Fay AM, Cummings SA, Burnett B, Tillmanns T. NSGC practice guideline: risk assessment and genetic counseling for hereditary breast and ovarian cancer. J Genet Couns 2013:22:155-163.

20. Cook-Deegan R, Conley JM, Evans JP, Vorhaus D. The next controversy in genetic testing: clinical data as trade secrets? Eur J Hum Genet 2013;21:585-588.

21. Genetic Alliance. Free the Data. 2013. http://www.free-the-data.org/. 2014 Accessed on 3 October 2014

22. Cummings $\mathrm{S}$. The genetic testing process: how much counseling is needed? J Clin Oncol 2000;18(suppl 1):60-64.
SCHERR et al | A preliminary investigation of genetic counselors' information needs

23. Group ALPCW. ACMG recommendations for standards for interpretation of sequence variations. Genet Med 2000;2:302-303.

24. Richards CS, Bale S, Bellissimo DB, et al.; Molecular Subcommittee of the ACMG Laboratory Quality Assurance Committee. ACMG recommendations for standards for interpretation and reporting of sequence variations: Revisions 2007. Genet Med 2008;10:294-300.

25. Robson M. Multigene panel testing: planning the next generation of research studies in clinical cancer genetics. J Clin Oncol 2014;32: 1987-1989. 